56 (21\%), and miscellaneous other disorders in 65 (24\%). The OBR confidence in their final diagnosis of RA was 7,5 $\pm 2,3$ (68/114 patients with a confidence of $8 / 10$ or more). A collegial diagnosis of certain $\mathrm{RA}(\mathrm{N}=57)$ or probable $\mathrm{RA}(\mathrm{N}=41)$ was made for 98 patients. The strength of OBR confidence in the diagnosis of RA was strongly correlated to that of the college $(r$ $=0,892)$. Conversely, the fulfilment of 4 ACR criteria for RA since the first visit was associated with an only slight increase in final OBR confidence $(7,9 \pm 2,1)$.

Conclusion Although only 13/114 (11\%) patients finally classified as RA changed from diagnosis during follow-up, the final confidence of OBR in their diagnosis of RA were below 8/10 in $40 \%$, and was only slightly improved by the fulfilment of ACR criteria. This emphasises the need for a close follow-up of these patients during the first years and the use of RA criteria for the classification of early arthritis.

\section{THU0137 LETHAL MEDIUM-VESSEL ARTERITIS MIMICKING DEEP SEPSIS FOLLOWING ETANERCEPT AND MINOCYCLINE THERAPY IN A PATIENT WITH RA}

JM Berthelot, J Glemarec, Y Maugars, A Prost. Rheumatology Unit, Hôtel-Dieu, Nantes, France

\subsection{6/annrheumdis-2001.1039}

\section{Background}

Objectives To report the onset of a severe and lethal mediumvessel panarteritis mimicking deep sepsis three weeks after etanercept withdrawal and minocycline therapy in a patient with RA.

\section{Methods}

Results A 50-year-old man with RA (7/7 1987 ACR criteria) had poorly responded to all DMARDs and low-dose steroids for 12 years. In September 1999, DAS-28 score of 6.8 led to a treatment by etanercept ( $25 \mathrm{mg}$ twice a week) for 4 months who remained poorly effective too (DAS-28 after 3 months: 6.05). Although no side-effects occurred, the patient wished to stop etanercept. A short cure by minocycline was administered. Ten days after, the patient's general condition worsened with fever (from $38^{\circ} \mathrm{C}$ to $39^{\circ} \mathrm{C}$ ), hyperleukocytosis $(24.109 / \mathrm{ml})$, confusion, pleural effusion, hypoalbuminemia (17.2 g/l), epigastric pain, and CRP levels up to $295 \mathrm{mg} / \mathrm{l}$. ANA and APL were negative, and ANCA only slightly positive (1/20, without anti-MPO antibodies). RF were 40 times the upper normal value. Extensive search for sepsis was unsuccessful. Highly false positive tests for myoglobin with ELISA kits using rodent antibodies were suggestive of anti-chimeric antibodies. The patient died in March 2000, three months after discontinuance of etanercept. An autopsy confirmed severe plurivisceral vasculitis typical of granulomatous, necrotising panarteritis, with both old and recent lesions involving mainly the medium-vessels in heart, muscles and gall-bladder.

Conclusion Although the relation of the patient's death to treatments is uncertain, a flare of rheumatoid vasculitis could have been involved. This severe vasculitis occurring soon after etanercept withdrawal and presenting first as unexplained fever mimicked deep sepsis for several weeks and misled the treatment strategy.

\section{THU0138 THE ACUTE PHASE RESPONSE DOES NOT FULLY EXPLAIN DYSLIPIDEMIA AND INSULIN RESISTANCE IN INFLAMMATORY ARTHRITIS}

${ }^{1} \mathrm{PH}$ Dessein, ${ }^{2} \mathrm{BI}$ Joffe, ${ }^{1} \mathrm{AE}$ Stanwix, ${ }^{3} \mathrm{Z}$ Moomal. ${ }^{1}$ Rheumatology; ${ }^{2}$ Carbohydrate and Lipid Metabolism Research Unit, Witwatersrand University/Johannesburg Hospital, Johannesburg; ${ }^{3}$ Statistics, National Research Foundation, Pretoria, South Africa

\subsection{6/annrheumdis-2001.1040}

Background An increased frequency of insulin resistance (IR) and dyslipidemia has been identified in inflammatory arthritis (IA). These metabolic disturbances may be attributable to disease activity and were implicated in the excess cardiovascular disease mortality as reported in IA.

Objectives In the present study we evaluated insulin sensitivity and lipid metabolism, as well as the potential role of the acute phase response as a predictor of these metabolic disturbances in IA.

Methods Eighty seven patients (38 rheumatoid arthritis, 29 spondyloarthropathy, 20 undifferentiated inflammatory arthritis) and 30 age-, gender- and race matched healthy controls $(\mathrm{CN})$ were enrolled. Patients who had taken glucocorticoids during the previous 2 months or were taking agents known to affect insulin sensitivity or lipid metabolism were excluded. The body mass index $\left(\mathrm{kg} / \mathrm{m}^{2}\right)$ was calculated in each subject. The erythrocyte sedimentation rate (ESR), plasma glucose, serum insulin and total cholesterol (chol), LDL-chol, HDL-chol and triglycerides were determined on fasting blood samples. IR was estimated by the homeostasis model assessment (HOMA). Results were expressed as mean (SD).

Results The BMI was 26.5 (4.2) in the patients and 22.9 (2.8) in the $\mathrm{CN}(\mathrm{p}<0.001)$. The HOMA (uU.mmol/ml.l) was 1.9 (1.3) in patients as compared to $1.1(0.5)$ in $\mathrm{CN}(\mathrm{p}=0.001)$. The HDL-chol was lower $(\mathrm{p}=0.002)$ and the total chol/HDL-chol was higher $(\mathrm{p}<0.001)$ in patients as compared to $\mathrm{CN}$. Only after controlling for the BMI in addition to the ESR, was the HOMA no longer different between patients and CN. The ESR and HOMA, but not the BMI, significantly predicted $(\mathrm{p}<0.05)$ high chol/HDL-chol ratios. However, after controlling for these variables, this lipid parameter remained different in patients as compared to $\mathrm{CN}$. Also, IA was associated with a positive family history for premature coronary artery disease $(p<0.03)$.

Conclusion Metabolic disturbances were identified in the majority of our patients and should therefore be addressed in the management of IA. IR could be attributed to the acute phase response and excess weight. However, dyslipidemia was associated with IA independent of the ESR, BMI and IR. Dyslipidemia may constitute a risk factor for IA.

\section{REFERENCES}

1 Svenson KL, et al. Metabolism 1988:37:125-30

2 Park YB, et al. J Rheumatol. 1999;26:1701-4

3 Dessein $\mathrm{PH}$, et al. Rheumatology 2001, in press

\section{THU0139 THE EFFECT OF INCREASED AIR PRESSURE ON PATIENTS WITH ACTIVE RHEUMATOID ARTHRITIS}

${ }^{1} \mathrm{LP}$ Robertson, ${ }^{2} \mathrm{~S}$ Marshall, ${ }^{2} \mathrm{P}$ Hickling. 'Department of Rheumatology, Royal Cornwall Hospital, Truro, UK; ${ }^{2}$ Department of Rheumatology, Derriford Hospital, Plymouth, UK 